\title{
The Three-Step Theory (3ST): A New Theory of Suicide Rooted in the "Ideation-to-Action" Framework
}

\author{
E. David Klonsky and Alexis M. May \\ Department of Psychology, University of British Columbia
}

\begin{abstract}
Klonsky and May (2014) argued that an "ideation-to-action" framework should guide suicide theory, research, and prevention. From this perspective, (a) the development of suicide ideation and (b) the progression from ideation to suicide attempts are distinct processes with distinct explanations. The present article introduces a specific theory of suicide rooted in the ideation-to-action framework: the Three-Step Theory (3ST). First, the theory hypothesizes that suicide ideation results from the combination of pain (usually psychological pain) and hopelessness. Second, among those experiencing both pain and hopelessness, connectedness is a key protective factor against escalating ideation. Third, the theory views the progression from ideation to attempts as facilitated by dispositional, acquired, and practical contributors to the capacity to attempt suicide. To examine the theory, the authors administered self-report measures to 910 U.S. adults utilizing Amazon's Mechanical Turk (oversampling for ideation and attempt histories). Results supported the theory's central tenets. First, an interactive model of pain and hopelessness accounted for substantial variance in suicide ideation. This result replicated in both men and women, and across age groups (i.e., 18-25, 26-35, and 36-70). Also as predicted, connectedness was most protective against ideation in those high on both pain and hopelessness. Finally, dispositional, acquired, and practical aspects of suicide capacity each predicted suicide attempt history over and above current and lifetime suicidal ideation. These initial findings support the 3ST. Implications for suicide prevention and future research are discussed.
\end{abstract}

Keywords: hopelessness, ideation, pain, suicide

Suicide is a leading cause of death worldwide, killing more than 800,000 people each year (World Health Organization [WHO], 2014a). A much larger number of people make suicide attempts, with some researchers estimating that approximately 25 attempts occur for every suicide death (Goldsmith, Pellmar, Kleinman, \& Bunney, 2002). An even greater number of people consider suicide; a world-

Address correspondence to E. David Klonsky, University of British Columbia, Department of Psychology, 2136 West Mall, Vancouver, BC V6T 1Z4, Canada; E-mail: EDKlonsky@gmail.com 
wide study found that for every person who attempts suicide, there are two to three who have seriously considered suicide without attempting it (Nock et al., 2008). Given this immense public health problem, suicide has been the focus of many research and prevention efforts, particularly in the past few decades. However, despite these efforts, there is no evidence of sustained reductions in suicide rates (WHO, 2014b). The development of more effective prevention and intervention strategies will very likely require a deeper understanding of the fundamental processes that cause suicide ideation, attempts, and deaths.

\section{TRADITIONAL THEORIES OF SUICIDE}

Many theorists have sought to explain suicide. For example, Shneidman (1985, 1993 ) explained suicide as a response to overwhelming pain (i.e., psychache), Durkheim (1897/1951) emphasized the role of social isolation, Baumeister (1990) described suicide as an escape from an aversive state of mind, and Beck and Abramson (Abramson et al., 2000; Beck, 1967) highlighted the role of hopelessness. These theories have been tremendously useful in guiding suicide research and prevention efforts. At the same time, these theories share a particular feature that may be limiting progress in understanding suicide: They fail to differentiate explanations for suicidal thoughts and suicidal behavior. This distinction is especially important when one considers that most people who develop suicidal ideation never go on to make a suicide attempt (Klonsky \& May, 2014; Nock et al., 2008).

\section{A NEW GENERATION OF THEORIES OF SUICIDE}

A critical advance in suicide theory occurred 10 years ago when Thomas Joiner (2005) introduced his Interpersonal Theory of Suicide. Joiner introduced a framework by which (a) suicidal ideation and (b) the progression from ideation to attempts were treated as separate processes that come with separate sets of explanations and risk factors. Joiner proposed a specific application of the framework: Perceptions of low belongingness and high burdensomeness combine to bring about desire for suicide, whereas high capability for suicide facilitates potentially lethal suicide attempts. However, we believe that the framework itself is at least as important a contribution to the field as the particulars of his theory. Inspired by Joiner's work, we recently proposed that an "ideation-to-action" framework should guide all suicide theory and research (Klonsky \& May, 2014). That is, the (a) development of suicide ideation and (b) progression from ideation to suicide attempts should be viewed as distinct processes with distinct explanations.

We view Joiner's Interpersonal Theory as the first ideation-to-action theory of suicide, which has likely spawned a new generation of suicide theories. For example, another recent theory of suicide, Rory O'Connor's (2011) Integrated Motivational-Volitional model, also proposes separate explanations for suicidal 


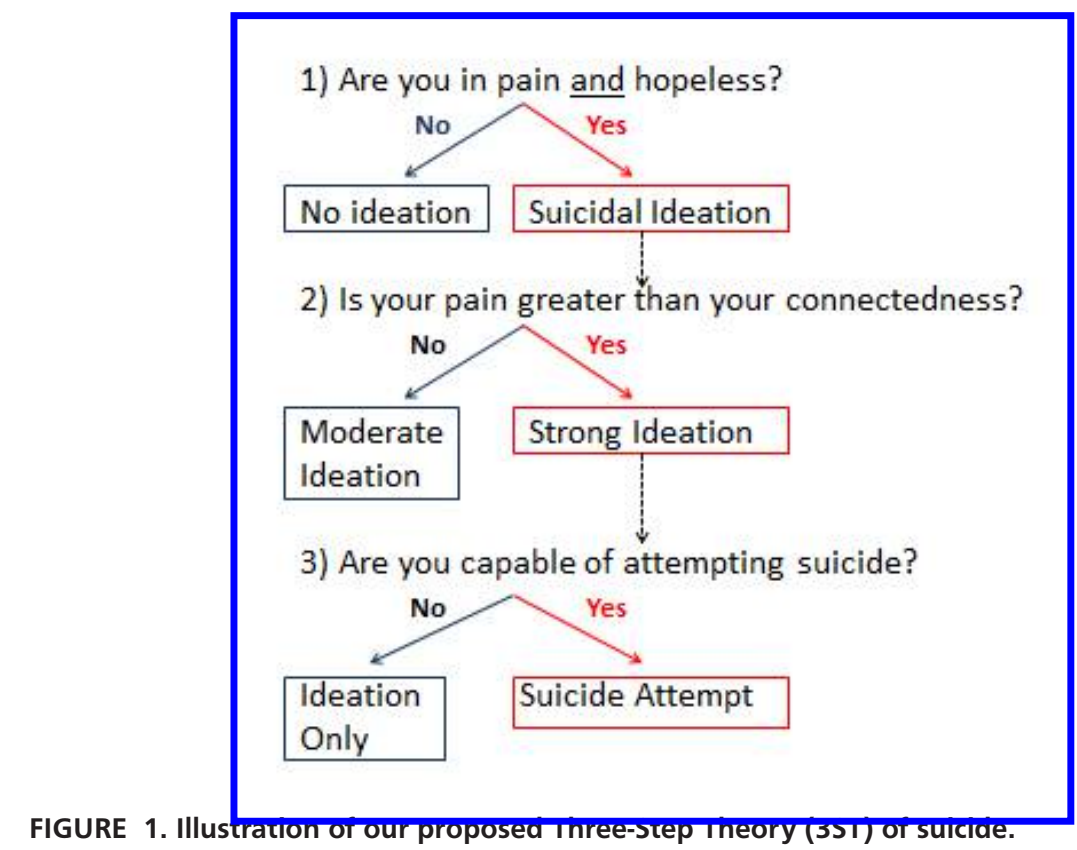

ideation and suicide attempts. O'Connor suggests that defeat and entrapment are the primary drivers of suicidal ideation, and that acquired capability along with others factors (e.g., access to lethal means, planning, impulsivity) explain the propensity to act on suicidal thoughts. We find it extremely encouraging that theorists appear to be embracing and utilizing an ideation-to-action framework.

\section{OUR PROPOSED THEORY OF SUICIDE}

The purpose of the present article is to describe and empirically evaluate our own theory of suicide positioned within an ideation-to-action framework. Like the theories of Joiner and O'Connor, our theory offers separate explanations for (a) the development of suicidal ideation and (b) the progression from suicidal ideation to attempts. The theory is relatively parsimonious in that suicide ideation and attempts are explained in terms of just four factors: pain, hopelessness, connectedness, and suicide capacity. The theory is illustrated in Figure 1 and explained here.

\section{STEP 1: DEVELOPMENT OF SUICIDAL IDEATION}

Regarding the development of suicidal ideation, we believe that the first step toward ideation begins with pain. Pain usually, but not necessarily, refers to psychological or emotional pain. Fundamentally, people are shaped by behavioral conditioning. We perform behaviors that are rewarded and avoid behaviors that are punished. If someone's day-to-day experience of living is characterized by pain, 
this individual is essentially being punished for living, which may decrease the desire to live and, in turn, initiate thoughts about suicide.

It is intentional that we do not specify the nature of the pain. Just as different sources of punishment can similarly shape behavior (e.g., electric shock [Alexander et al., 1973], loud noises [Watson \& Rayner, 1920], noxious odors [Tanner \& Zeiler, 1975], social exclusion [Williams, Cheung, \& Choi, 2000], or any sufficiently aversive stimulus [Mazur, 2012]), we believe that different sources of pain can all lead to a decreased desire to live. These may include physical suffering (Ratcliffe, Enns, Belik, \& Sareen, 2008), social isolation (Durkheim, 1897/1951), burdensomeness and low belongingness (Joiner, 2005), defeat and entrapment (O'Connor, 2011), negative self-perceptions (Baumeister, 1990), and myriad other aversive thoughts, emotions, sensations, and experiences. The first step toward suicidal ideation begins with pain, regardless of its source.

However, pain alone is not sufficient to produce suicidal ideation. If someone living in pain has hope that the situation can improve, the individual likely will focus on obtaining a future with diminished pain rather than on the possibility of ending his or her life. For this reason, hopelessness is also required for the development of suicidal ideation. From our perspective, when someone's day-to-day experience is characterized by pain, and the person feels hopeless that the pain will improve, he or she will consider suicide. In short, the combination of pain and hopelessness is what causes suicide ideation to develop. Our perspective is consistent with recent research finding that pain and hopelessness are the two most common motivations for suicide attempts (May \& Klonsky, 2013).

Importantly, we believe it is the combination of pain and hopelessness that is required to bring about suicidal ideation. Someone in pain but with hope for a better future will continue to engage with life. Similarly, someone who feels hopeless about the future but without day-to-day pain will not consider suicide. The latter situation may seem less intuitive than the former, so consider the following example. A young adult recently graduated from university and moved back in with her parents. If this young adult lacks a marketable degree, good grades, and a sense of her career interests, she may very well feel hopeless about the future. However, as long as her day-to-day experience remains comfortable with little or no pain-for example, if her food and shelter are provided, she has free time to spend with friends, and she enjoys participating in activities of her choosing-she is unlikely to consider suicide. Pain and hopelessness in combination are required for the development of suicidal ideation.

\section{STEP 2: STRONG VERSUS MODERATE IDEATION}

The second step toward potentially lethal suicidal behavior involves connectedness. Connectedness most often means connection to other people; however, we use the term more broadly. Connectedness can also refer to one's attachment to a job, project, role, interest, or any sense of perceived purpose or meaning that keeps one invested in living. Connectedness matters, because even if someone feels pain and hopelessness and considers suicide, the suicidal ideation will remain moder- 
ate (e.g., "sometimes I think I might be better off dead") rather than strong (e.g., "I would kill myself if I had the chance") as long as one's connectedness to life is greater than one's pain. Consider the example of a parent who experiences daily pain and hopelessness, but is invested in or connected to his or her children. If the parent's connectedness is greater than the parent's pain, this individual may still have passive ideation but will not progress to active desire for suicide. However, if both pain and hopelessness are present, and connectedness is absent or less than the pain, the individual will have strong suicidal ideation and an active desire to end his or her life.

Disrupted connectedness is similar to low belongingness and burdensomeness as described in Joiner's Interpersonal Theory. We therefore want to highlight a key difference between our theory and the Interpersonal Theory. In our theory, the primary role of connectedness is to protect against strong suicidal ideation in those at high risk due to pain and hopelessness. While we believe disrupted connectedness can contribute directly to pain and hopelessness, we do not view it as necessary for the development of pain or hopelessness, and thus the development of suicidal ideation. We believe that many people with disrupted connectedness do not have suicidal ideation, and that many people with suicidal ideation do not experience disrupted connectedness.

To be clear, our emphasis on pain, hopelessness, and connectedness in explaining suicidal ideation is not meant to suggest that other traditional risk factors for suicide are irrelevant. Indeed, we believe that numerous disorders (e.g., depression), states of mind (e.g., self-criticism), personality traits (e.g., borderline personality), temperaments/dispositions (e.g., negative emotionality), and experiences (e.g., interpersonal loss) are highly relevant to suicidal ideation. However, we believe that they are relevant in a specific way, through their effects on pain, hopelessness, and/or connectedness. For example, we would expect depression to relate to suicidal ideation to the extent that it influences pain, hopelessness, and/or connectedness, but not beyond.

\section{STEP 3: PROGRESSION FROM IDEATION TO ATTEMPTS}

Once an individual has developed a desire to end his or her life, the next question is whether the person will act on that desire and make an attempt. We agree with Joiner (2005) that the key determinant is whether the individual has the capability to make a suicide attempt. As Joiner argues, people are biologically and evolutionarily wired to avoid pain, injury, and death. It is therefore very difficult for people to attempt suicide, even in the presence of strong suicidal ideation. However, we expand on Joiner's notion of capability in two ways.

Joiner emphasizes acquired capability. Acquired capability refers to an individual's habituation to pain, fear, and death through exposure to life experiences such as physical abuse, nonsuicidal self-injury, the suicide of a family member or friend, combat training, or any other experience that subjects someone to painful and provocative events. We take a broader perspective and propose three specific 
categories of variables that contribute to suicide capacity: dispositional, acquired, and practical.

Dispositional refers to relevant variables that are driven largely by genetics, such as pain sensitivity (Young, Lariviere, \& Belfer, 2012) or blood phobia (Czajkowski, Kendler, Tambs, Røysamb, \& Reichborn-Kjennerud, 2011). For example, someone born with low pain sensitivity will have a higher capacity to carry out a suicide attempt, whereas someone born with a squeamishness or even phobia of blood will have a lower capacity. Indeed, more recent work from Joiner and others has found that capability for suicide is largely genetic (Smith et al., 2012). Acquired refers to the same construct Joiner describes, that habituation to experiences associated with pain, injury, fear, and death can lead over time to higher capacity for a suicide attempt. Practical refers to concrete factors that make a suicide attempt easier. There are many kinds of practical factors. For example, someone with both knowledge of and access to lethal means, such as a firearm, will be more able to act on suicidal thoughts than someone who lacks knowledge of and access to lethal means. Another example is anesthesiologists and other medical professionals whose suicide rates are elevated (Swanson, Roberts, \& Chapman, 2003). Our theory suggests that suicide rates are elevated because these individuals have both extensive knowledge of how to end one's life painlessly and easy access to the necessary drugs. In summary, dispositional, acquired, and practical factors contribute to the capacity for attempted suicide, and an individual with strong suicidal ideation will only make a suicide attempt if and when they have the capacity to do so.

\section{THE PRESENT STUDY}

The present study conducts an initial empirical evaluation of our proposed theory's key principles in a large, sociodemographically diverse sample of adults in the United States. The key hypotheses we examined were:

(1) Pain and hopelessness will interact to predict current suicidal ideation (and predict suicidal ideation better than a comparison model consisting of burdensomeness and low belongingness).

(2) Connectedness protects against escalation of suicidal ideation among those high on both pain and hopelessness.

(3) Dispositional, acquired, and practical contributors to suicide capacity will distinguish lifetime suicide attempters from those with histories of ideation but not attempts.

\section{METHODS}

\section{PARTICIPANTS AND PROCEDURE}

The data for this article were taken from a larger study on suicide ideation and attempts. The study was advertised on Mechanical Turk, a crowdsourcing Internet 
marketplace in which participants see descriptions of tasks they may complete, estimated time required, and compensation amount. The study consisted of a screening questionnaire and a full battery of questionnaires, both completed online. All participants filled out the screening questionnaire, which included questions about suicide ideation and attempt history. In the first wave of data collection, all respondents were also invited to complete the full survey, regardless of suicidality history $(n=906)$. In the second phase of the study, participants were invited to complete the full survey only if they endorsed a history of suicide ideation or attempt on the screening questionnaire $(n=191)$. The full survey included all the measures described here, as well as four validity questions to ensure that participants were attending to the questionnaire appropriately. After we removed participants who failed to answer the validity questions correctly, completed the survey multiple times, or failed to complete the suicide ideation measure, 910 participants remained.

Approximately half of the participants were male (53\%) with a mean age of 31 years $(S D=10$; range $=18-70)$. Regarding ethnicity, the sample was predominantly Caucasian (76\%). The remaining participants were African American (6\%), Asian American (8\%), Latino/Hispanic (4\%), multiple ethnicities (4\%), and other $(2 \%)$. Fifteen percent of the sample endorsed a minority sexual orientation (e.g., gay, bisexual, lesbian, questioning). Most participants reported that their highest education level was either graduating from college $(38 \%)$ or taking some college classes $(36 \%)$. Of the remaining participants, $12 \%$ reported a high school degree or less and 14\% reported some graduate school or more. The sample was drawn from diverse regions of the United States: Southeast (25\%), Far West (19\%), Mid-Atlantic (17\%), Great Lakes (16\%), Southwest (10\%), Plains (5\%), New England (5\%), and Rocky Mountains (4\%).

Regarding history of suicide, in the unselected sample, $71 \%$ reported no suicidality history, $19 \%$ reported a history of ideation or planning but no attempt, and $10 \%$ reported a previous suicide attempt. In the full sample, which oversampled for histories of suicidality and was the basis for all the analyses reported here, $59 \%$ reported no suicidality history, $27 \%$ reported a history of ideation or planning but no attempt, and $14 \%$ reported a previous suicide attempt.

\section{MEASURES}

Suicidality Screening. Three items from the Youth Risk Behavior Surveillance Survey (YRBS; Centers for Disease Control, 2009) were used to screen for a lifetime history of suicide ideation, planning, or attempt. These questions have good to excellent reliability and validity (Brener et al., 2002; May \& Klonsky, 2011).

Current Suicide Ideation. Current suicide ideation was assessed using the selfreport version of the Beck Scale for Suicide Ideation (BSS; Beck \& Steer, 1991) and the suicide ideation item from the Beck Depression Inventory (BDI; Beck, Rush, Shaw, \& Emery, 1979). The BSS is a widely used 19-item measure of suicide ideation. It covers active and passive ideation, including the presence of plans or preparation. Each item has three response options, which are scored from 0 
to 2. Thus, total BSS scores could range from 0 to 38. The BDI item has four response options ranging from no ideation to active ideation. If participants endorsed nonzero ideation on any of the first five BSS items or the BDI item, they completed the entire BSS and were assigned the summed BSS total as their score for suicide ideation. Otherwise, they were assigned an ideation score of 0 . In our sample, reliability of the BSS was excellent $(\alpha=.86)$.

Psychological Pain. The 13-item Scale of Psychache measured current emotional or mental pain as conceptualized by Edwin Shneidman (1993). Items are rated on a 5-point Likert scale and total scores can range from 13 to 65 . The psychometric properties of this measure have been established (Holden, Mehta, Cunningham, \& McLeod, 2001). In this sample, reliability was excellent $(\alpha=.97)$.

Hopelessness. The Beck Hopelessness Scale-Short Form (BHS-SF; Beck, Weissman, Lester, \& Trexler, 1974) assessed hopelessness within the past week. Items were rated as true or false and total scores can range from 0 to 4 . The BHSSF is a 4-item version of the widely used Beck Hopelessness Scale (Beck et al., 1974), and the short form has demonstrated good psychometric properties (Aish, Wasserman, \& Renberg, 2001; Yip \& Cheung, 2006). In this sample, reliability was excellent $(\alpha=.86)$.

Burdensomeness and Belongingness. These constructs, which are part of the Interpersonal Psychological Theory, were measured with the 10 -item version of the Interpersonal Needs Questionnaire (INQ; Bryan, 2011; Hill et al., 2014; Van Orden, Witte, Gordon, Bender, \& Joiner, 2008). The INQ was designed to measure participants' beliefs about the extent to which they feel connected to others (i.e., belongingness) and the degree to which they feel they are a burden to others (i.e., burdensomeness). Participants indicate the degree to which each item is true for them on a 7-point Likert scale, and scores for each factor can range from 5 to 35 .

Suicide Capacity. Two measures were used to assess suicide capacity. First, the 20-item Acquired Capability for Suicide Scale (ACSS; Van Orden et al., 2008) measured the extent to which individuals perceive themselves as able to perform dangerous tasks and their degree of comfort with potentially dangerous situations. Items were rated on a 5-point Likert scale, and scores can range from 0 to 80 .

Second, a six-item Suicide Capacity Scale (SCS-3) designed by our lab assessed three characteristics that we believe contribute to suicide capacity: Dispositional Capacity (i.e., long-standing pattern of low fear of pain or death), Acquired Capacity (i.e., fear of pain or death had decreased over time), and Practical Capacity (i.e., access to and knowledge of suicide methods). Items were rated on a 7-point Likert scale. Total scores for the SCS-3 could range from 0 to 36 . The correlations of this measure and its three subscales with the ACSS are included in Table 1.

\section{RESULTS}

Descriptive statistics and intercorrelations for the key study variables are presented in Table 1. Twenty-one percent of the sample reported current ideation. Among 


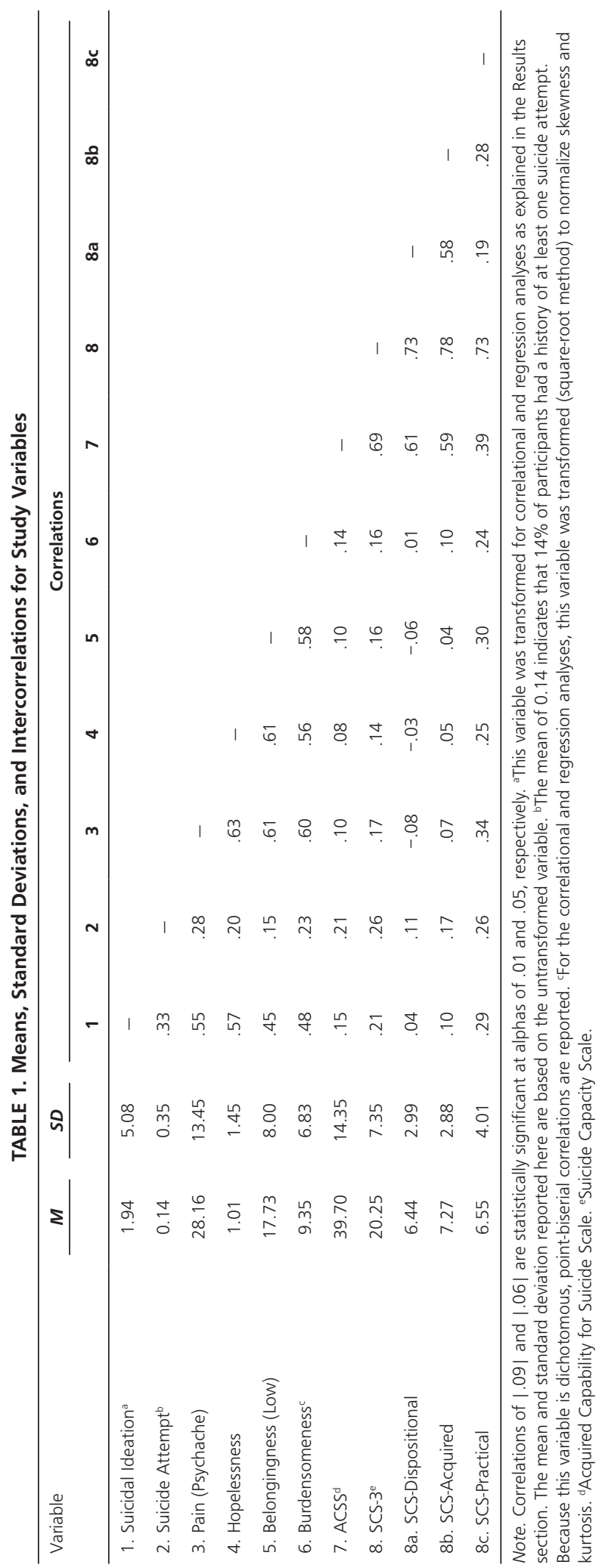


those participants reporting at least some ideation, the mean of the untransformed BSS score was $10.8(S D=6.9)$. Fourteen percent of participants reported at least one suicide attempt. Here we describe results addressing each of our key hypotheses regarding the development of suicide ideation and attempts.

\section{HYPOTHESIS 1: PAIN AND HOPELESSNESS INTERACT TO PREDICT SUICIDAL IDEATION}

Because many participants had scores of 0 on suicidal ideation, we transformed the variable by repeatedly taking its square root (three times total) until skewness and kurtosis were reduced to acceptable levels. The transformed variable was used in all subsequent analyses. In addition, we centered the pain and hopelessness variables to facilitate interpretation of their interactive effect on suicidal ideation. These transformed/centered variables were utilized for subsequent regression analyses.

First, we report the direct effects of pain and hopelessness on suicidal ideation. As expected, both pain $(r=.55)$ and hopelessness $(r=.57)$ exhibited robust correlations with suicidal ideation. Not surprisingly, pain and hopelessness also related strongly with one another $(r=.63)$.

Second, as a direct test of our first hypothesis, we examined whether pain and hopelessness interacted to predict suicidal ideation. The interaction term was significant $(t=6.35, p<.001)$, and the full model accounted for $41 \%$ of the variance in suicidal ideation. The interaction term itself explained an additional $3 \%$ of variance over and above the main effects. However, because the interaction term correlates strongly with both main effect terms, the $3 \%$ of unique variance added may not fully capture the interactive nature of pain and hopelessness in predicting suicidal ideation. Thus, to illustrate the potential clinical significance of this pattern, median splits were utilized to create low and high subgroups for pain and hopelessness. As can be seen in Figure 2, suicidal ideation is negligible in subgroups with (a) low pain and hopelessness or (b) either high pain or high hopelessness, but is substantially higher in the subgroup (c) reporting both high pain and high hopelessness. In addition, we examined the percentage of participants meeting an empirically derived cutoff for severe suicidal ideation (i.e., scores of 24 or above on the BSS; Cochrane-Brink, Lofchy, \& Sakinofsky, 2000) who fell into each of these three subgroups. This high threshold for severe suicidal ideation was met by 12 participants in our sample; notably, all 12 fell into the subgroup high on both pain and hopelessness.

Next, we examined whether the statistically significant interaction between pain and hopelessness would appear consistently across demographic subgroups. It did. The interaction was statistically reliable in both men $(t=4.28, p<.001)$ and women $(t=4.45, p<.001)$, as well as in participants ages $18-25(t=3.80$, $p<.001), 26-35(t=4.55, p<.001)$, and 36-70 $(t=2.31, p=.02)$.

Finally, we wanted to evaluate our model of suicidal ideation in comparison to an established, well-researched model. We therefore also examined the variables hypothesized by the Interpersonal Theory of Suicide (Joiner, 2005; Van 


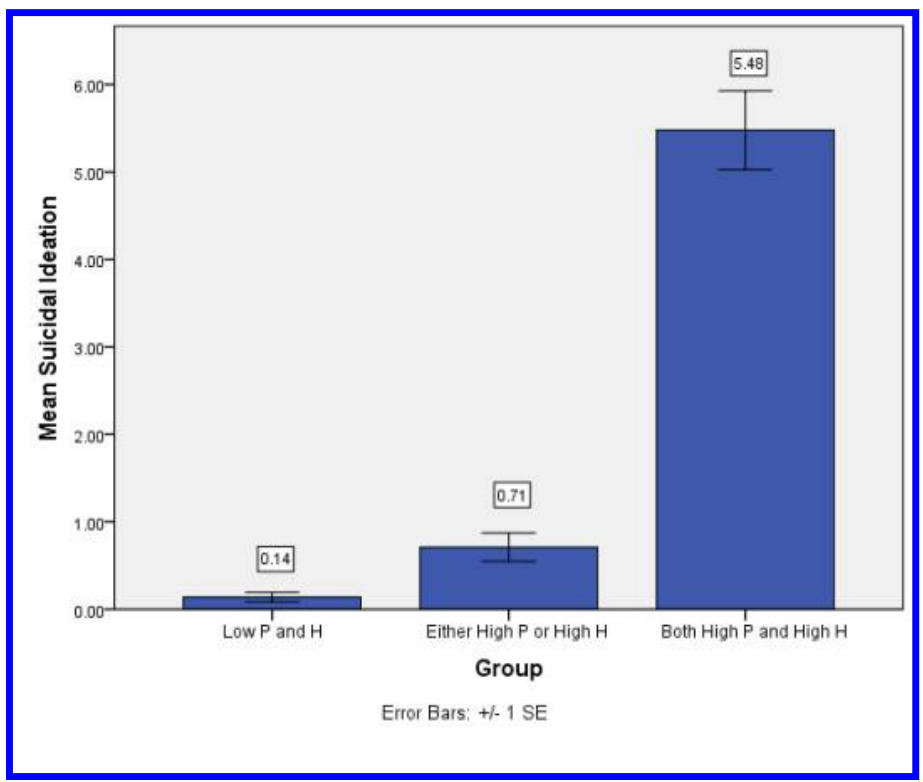

FIGURE 2. Interactive effects of pain (P) and hopelessness $(\mathrm{H})$ on suicidal ideation.

Orden et al., 2010) to explain suicidal ideation. This comparison model specifies that perceptions of low belongingness and high burdensomeness interact to explain suicidal ideation. In the current sample, belongingness and burdensomeness indeed interacted significantly to predict suicidal ideation $(t=4.6, p<.001)$; however, the full model including the interaction term accounted for only $30 \%$ of the variance in suicidal ideation-lower than the $41 \%$ reported earlier for our hypothesized model.

\section{HYPOTHESIS 2: CONNECTEDNESS PROTECTS AGAINST ESCALATION OF SUICIDAL IDEATION IN INDIVIDUALS WITH BOTH PAIN AND HOPELESSNESS}

Our theory hypothesizes that connectedness can foster a desire to live even among those with both pain and hopelessness, and thus protect against higher levels of ideation among this at-risk subgroup. We conducted two analyses to evaluate this hypothesis. First, we examined whether the relationship of connectedness to suicidal ideation is particularly strong in the subgroup of participants high on both pain and hopelessness. We utilized the belongingness scale of the INQ (Bryan, 2011; Van Orden et al., 2008) to assess connectedness. As predicted, there was a significant interaction between connectedness and pain-hopelessness status $(t$ $=6.28, p<.001)$. In particular, the relationship of connectedness with suicidal ideation was $r=.36$ in the subgroup with both high pain and high hopelessness $(n=283)$, compared to just $r=.14$ in everyone else $(n=627)$.

Second, we wanted to test the more specific hypothesis that suicidal ideation is buffered in individuals with combined pain and hopelessness when connect- 
edness exceeds pain (i.e., when one's connection to someone/something in life exceeds one's pain). To do this, we standardized scores for pain and connectedness, and then subtracted connectedness scores from pain scores. Thus, positive scores indicated that pain exceeds connectedness, whereas negative scores indicated that connectedness exceeds pain. If our hypothesis is correct, this difference score should be a particularly powerful predictor of suicidal ideation in the combined pain and hopelessness subgroup, and less predictive of suicidal ideation in everyone else. As predicted, the interaction between the pain-connectedness difference score and group status (i.e., combined high pain and high hopelessness vs. everyone else) was statistically significant $(t=8.07, p<.001)$. In particular, the correlation of suicidal ideation with the pain-connectedness difference score was stronger $(r=.47)$ for the high pain and high hopelessness subgroup compared to everyone else $(r=.22)$.

\section{HYPOTHESIS 3: DISPOSITIONAL, ACQUIRED, AND PRACTICAL CONTRIBUTORS TO CAPACITY FOR SUICIDE PREDICT TRANSITION FROM IDEATION TO ATTEMPTS}

Because there was no preexisting measure of the three components of suicide capacity stipulated in our theory (dispositional, acquired, and practical), we developed our own measure for the purposes of this study: the Suicide Capacity Scale (SCS-3). To help verify the construct validity of this measure, we examined its correlation with the ACSS, an established measure of capability for suicide (Van Orden et al., 2008). The ACSS includes items relevant to the broad construct of suicide capability but does not specifically target or distinguish dispositional, acquired, and practical aspects. In support of our measure's validity, its correlation with the ACSS was positive and large $(r=.69, p<.001)$. In addition, all three subscales correlated positively with the ACSS: dispositional $(r=.62)$, acquired $(r$ $=.59)$, and practical $(r=.39)$ (all $p$ s $<.001)$.

Next, as a direct test of the hypothesis, $t$ tests were utilized to compare dispositional, acquired, and practical components of suicide capacity between (a) participants with histories of suicide ideation but not attempts $(n=246)$ and (b) participants with histories of suicide attempts $(n=127)$. All three contributors to suicide capacity differentiated the ideation and attempt subgroups: dispositional $($ Cohen's $d=.34, p<.001)$, acquired $(d=.38, p<.001)$, and practical $(d=.23$, $p<.02$ ). In addition, total capacity (i.e., the sum of the three subscales) distinguished suicide ideators and attempters: $d=.42, p<.001$. Considering that the SCS-3 consists of just six items compared to 20 items for the ACSS, this effect size compares favorably with that produced when comparing the ACSS between ideators and attempters $(d=.44)$. Finally, logistic regressions revealed that all three SCS-3 subscales maintain their associations with attempt history even when controlling for current suicidal ideation $(p s \leq .001$ for dispositional and acquired, $p=.03$ for practical). 


\section{DISCUSSION}

Thomas Joiner's Interpersonal Theory of Suicide spawned a new generation of suicide theories that utilize an ideation-to-action framework. These theories regard the development of suicidal ideation and the progression from ideation to attempts as distinct processes that require distinct explanations. The purpose of the present article was to describe a new ideation-to-action theory of suicide and report findings from an initial empirical evaluation of the theory. The primary tenets of the Three-Step Theory (3ST) are that (a) suicidal ideation develops due to a combination of pain and hopelessness, (b) connectedness is a key protective factor against escalating ideation in those high on both pain and hopelessness, and (c) progression from suicide ideation to attempts occurs when dispositional, acquired, and practical factors create sufficiently high capacity to face the pain and fear inherent in attempting to end one's life. Findings from a large sample of United States adults provide relatively strong support for the theory, although it will be essential to replicate these findings and examine the theory in other populations.

The first tenet, that suicide ideation is driven by the combination of pain and hopelessness, was strongly supported. Pain and hopelessness interacted to predict suicidal ideation, and this interaction replicated across different genders and age groups. Moreover, the model including pain, hopelessness, and their interaction explained more variability in suicidal ideation than a comparison model including belongingness, burdensomeness, and their interaction. Results are consistent not only with our theory, but also with recent research findings that suicide attempts are motivated by pain and hopelessness more than by any other factor (May \& Klonsky, 2013). We also found evidence for the second tenet: that connectedness plays a particularly important protective role against suicidal ideation in those at greatest risk for ideation (i.e., in those high on both pain and hopelessness). In particular, connectedness, as well as the degree to which participants' connectedness exceeded their pain, predicted lower suicidal ideation among those with combined pain and hopelessness, but relatedly minimally to suicidal ideation for everyone else.

Taken together, the results described here support our theory of how pain, hopelessness, and disrupted connectedness work in concert to bring about suicidal ideation. It is useful to address how our theory is different from previous models emphasizing pain (Shneidman, 1993), hopelessness (Abramson et al., 2000), and connectedness (Joiner, 2005). One key difference from Shneidman and Abramson et al. is that we believe the combination of pain and hopelessness is what matters. The importance of the combination can be viewed in Figure 2, which shows that suicidal ideation is negligible both in subgroups with (a) neither pain nor hopelessness and (b) either pain or hopelessness; in contrast, the subgroup with combined pain and hopelessness is where elevated suicidal ideation can be found. A second key difference regards the role of connectedness, which is similar to the belongingness and burdensomeness constructs emphasized by Joiner (2005). Joiner suggests that suicidal ideation is driven by feelings of low belongingness and high burdensomeness, specifically when one feels hopeless that these feelings will change (Van 
Orden et al., 2010). While we believe that belongingness and burdensomeness can contribute to the pain and hopelessness that drive suicidal ideation, we do not believe they are necessary for pain, hopelessness, or suicidal ideation. Instead, we view connectedness as playing an important protective role in those at risk for strong/intense suicidal ideation due to pain and hopelessness. Finally, we recognize that hundreds of suicide correlates have been identified and that many have been conceptualized as important risk factors (American Association of Suicidology, 2013). Our theory does not dispute the relevance of these factors; rather, it offers a framework for understanding them. From our perspective, the relevance to suicide of any given correlate or risk factor can be understood in terms of its contributions to pain, hopelessness, connectedness, and/or suicide capacity.

The third key tenet of our theory was also supported. This tenet proposes that dispositional, acquired, and practical contributors to suicide capacity explain the progression from suicide ideation to attempts. In the present study, subscales measuring each of these three contributors were found to be higher in those with a history of attempted suicide than in those with a history of suicide ideation only. Associations were similar in magnitude for each of the contributors to suicide capacity. This pattern is consistent with Joiner's (2005) emphasis on acquired capability, but also supports the relevance of dispositional and practical contributors to suicide capacity. At the same time, it is worth noting that the associations between suicide capacity and suicide attempt history were relatively small, and thus explain only a small amount of variability in the progression from suicide ideation to attempts. It will be critical for future research to continue to clarify how and when suicidal ideation leads to suicidal behavior.

Our theory has potentially important implications for suicide prevention and intervention. Our theory suggests specific targets for reducing suicide risk. If we want to reduce suicide risk, we can (a) reduce pain, (b) increase hope, (c) improve connectedness, and/or (d) reduce capacity. The manner in which these variables are targeted cannot be stipulated because prevention and intervention can look very different depending on setting (e.g., school vs. treatment center), age (e.g., adolescents vs. older adults), and level of intervention (e.g., individual psychotherapy vs. government policy) among many other factors. For example, all four might be useful targets at the level of the individual (e.g., psychotherapy), and the fourth might also be a useful target at the level of government policy (e.g., bridge barriers, increased controls on firearms) (see Miller, Azrael, \& Barber, 2012). These considerations regarding context notwithstanding, we believe that any effort to prevent or treat suicidality should target one or more of these four factors and will succeed to the extent that one or more of these four factors is changed for the better.

This article represents an initial description and empirical evaluation of the 3ST. There are several important limitations and future directions. First, we examined our theory in a nonclinical population. Even though we oversampled for participants with histories of suicide ideation and attempts, our sample likely had less severe psychopathology and suicidal ideation as compared to an outpatient or inpatient sample. Future research should evaluate our theory in clinical settings. Sec- 
ond, our study focused on adults, and primarily on adults between the ages of 18 and 35. Future research should examine the relevance of our theory in adolescent and older adult populations. Third, our study relied on self-report measures. We utilized measures with good psychometric properties, but there are certain biases inherent in self-report methods, and future research should determine whether results replicate when using interview and behavioral measures. Fourth, the data we obtained are cross-sectional. Longitudinal data will be important for determining whether the variables we emphasize are best conceptualized as contributors to, rather than consequences of, suicide ideation and attempts. Longitudinal data will be especially useful for examining whether higher suicide capacity predicts transition to suicidal behavior among those with suicidal ideation. In addition, longitudinal and diary methodologies can help clarify the time scales at which the factors we emphasize lead to suicidal ideation and attempts. For example, must pain and hopelessness develop gradually over time before they lead to suicidal ideation, or can they develop and lead to suicidal ideation in time frames as short as a week, a day, or even an hour? As recently emphasized by Glenn and Nock (2014), it will be important to increase the methodological creativity and sophistication of our studies in order to obtain a more accurate, nuanced, and actionable understanding of suicide.

\section{REFERENCES}

Abramson, L. M., Alloy, L. B., Hogan, M. E., Whitehouse, W. G., Gibb, B. E., Hankin, B. L., \& Cornette, M. M. (2000). The hopelessness theory of suicidality. In T. Joiner \& M. D. Rudd (Eds.), Suicide science: Expanding the boundaries (pp. 17-32). Norwell, MA: Kluwer Academic Publishers.

Aish, A. M., Wasserman, D., \& Renberg, E. S. (2001). Does Beck's Hopelessness Scale really measure several components? Psychological Medicine, 31, 367-372.

Alexander, A. B., Chai, H., Creer, T. L., Miklich, D. R., Renne, C. M., \& de A. Cardoso, R. R. (1973). The elimination of chronic cough by response suppression shaping. Journal of Behavior Therapy and Experimental Psychiatry, 4, 75-80.

American Association of Suicidology. (2013). Risk factors for suicide. Retrieved November 14, 2014, from http://www.suicidology.org/ Portals/14/docs/Resources/FactSheets/RiskFactors_2013.pdf

Baumeister, R. F. (1990). Suicide as escape from self. Psychological Review, 97, 90-113.

Beck, A. T. (1967). Depression: Clinical, experimental, and theoretical aspects. New York, NY: Harper \& Row.

Beck, A. T., Rush, A. J., Shaw, B. F., \& Emery, G. (1979). Cognitive therapy of depression: $A$ treatment mamual. New York, NY: Guilford Press.
Beck, A. T., \& Steer, R. A. (1991). Manual for Beck scale for suicide ideation. San Antonio, TX: Psychological Corporation.

Beck, A.T., Weissman, A., Lester, D., \& Trexler, L. (1974). The measurement of pessimism: The Hopelessness Scale. Journal of Consulting and Clinical Psychology, 47, 861-863.

Brener, N. D., Kann, L., McManus, T., Kinchen, S. A., Sundberg, E. C., \& Ross, J. G. (2002). Reliability of the 1999 Youth Risk Behavior Survey questionnaire. Journal of Adolescent Health, 31, 336-342.

Bryan, C. J. (2011). The clinical utility of a brief measure of perceived burdensomeness and thwarted belongingness for the detection of suicidal military personnel. Journal of Clinical Psychology, 67, 981-992.

Centers for Disease Control and Prevention. (2009). Youth Risk Behavior Survey. Retrieved November 10, 2014, from http:// www.cdc.gov/HealthyYouth/yrbs/questionnaire rationale.htm

Cochrane-Brink, K. A., Lofchy, J. S., \& Sakinofsky, I. (2000). Clinical rating scales in suicide risk assessment. General Hospital Psychiatry, 22, 445-451.

Czajkowski, N., Kendler, K. S., Tambs, K., Røysamb, E., \& Reichborn-Kjennerud, T. (2011). The structure of genetic and environmental risk factors for phobias in wom- 
en. Psychological Medicine, 41, 1987-1995. doi:10.1017/S0033291710002436.

Durkheim, E. (1951). Suicide: A study in sociology. New York, NY: Free Press. (Original work published 1897)

Glenn, C. R., \& Nock, M. K. (2014). Improving the short-term prediction of suicidal behavior. American Journal of Preventive Medicine, 47(3, Suppl. 2), S176-S180. doi:10.1016/j. amepre.2014.06.004

Goldsmith, S. K., Pellmar, T. C., Kleinman, A. M., \& Bunney, W. E. (Eds.). (2002). Reducing suicide: A national imperative. Washington, DC: National Academy Press.

Hill, R. M., Rey, Y., Marin, C. E., Sharp, C., Green, K. L., \& Pettit, J. W. (2014). Evaluating the Interpersonal Needs Questionnaire: Comparison of the reliability, factor structure, and predictive validity across five versions. Suicide and Life-Threatening Behavior. Advance online publication. doi:10.1111/sltb.12129

Holden, R. R., Mehta, K., Cunningham, J., \& McLeod, L. D. (2001). Development and preliminary validation of a scale of psychache. Canadian Journal of Behavioural Science, 33, 224-232.

Joiner, T. E. (2005). Why people die by swicide. Cambridge, MA: Harvard University Press.

Klonsky, E. D., \& May, A. M. (2014). Differentiating suicide attempters from suicide ideators: A critical frontier for suicidology research. Suicide and Life-Threatening Behavior, 1, 1-5.

May, A., \& Klonsky, E. D. (2011). Validity of suicidality items from the Youth Risk Behaviors Survey in a high-school sample. Assessment, $18,379-381$.

May, A. M., \& Klonsky, E. D. (2013). Assessing motivations for suicide attempts: Development and psychometric properties of the Inventory of Motivations for Suicide Attempts (IMSA). Suicide and Life-Threatening Behavior, 43, 532-546.

Mazur, J. E. (2012). Learning and behavior (7th ed.). Englewood Cliffs, NJ: Prentice-Hall.

-Miller, M., Azrael, D., \& Barber, C. (2012). Suicide mortality in the United States: The importance of attending to method in understanding population-level disparities in the burden of suicide. Anmual Review of Public Health, 33, 393-408.

-Nock, M. K., Borges, G., Bromet, E. J., Alonso, J., Angermeyer, M., Beautrais, A., . . . Williams, D. (2008). Cross-national prevalence and risk factors for suicidal ideation, plans and attempts. British Journal of Psychiatry, 192, 98-105.

O'Connor, R. C. (2011). Towards an integrated motivational-volitional model of suicidal behaviour. In R. C. O’Connor, S. Platt, \& J. Gordon (Eds.), International handbook of suicide prevention: Research, policy, and practice (pp. 181-198). Malden, MA: John Wiley \& Sons.

Ratcliffe, G. E., Enns, M. W., Belik, S. L., \& Sareen, J. (2008). Chronic pain conditions and suicidal ideation and suicidal attempts: An epidemiological perspective. Clinical Journal of Pain, 24, 204-210.

Shneidman, E. S. (1985). Definition of suicide. New York, NY: Wiley

Shneidman, E. S. (1993). Suicide as psychache: A clinical approach to self-destructive behavior. Northfield, NJ: Jason Aronson.

Smith, A. R., Ribeiro, J., Mikolajewski, A., Taylor, J., Joiner, T., \& Iacono, W. G. (2012). An examination of environmental and genetic contributions to the determinants of suicidal behavior among male twins. Psychiatry Research, 197, 60-65.

Swanson, S. P., Roberts, L. J., \& Chapman, M. D. (2003). Are anaesthetists prone to suicide? A review of rates and risk factors. Anaesthesia and Intensive Care, 31, 434-445.

Tanner, B. A., \& Zeiler, M. (1975). Punishment of self-injurious behavior using aromatic ammonia as the aversive stimulus. Journal of Applied Behavior Analysis, 8, 53-57.

Van Orden, K. A., Witte, T. K., Cukrowicz, K. C., Braithwaite, S. R., Selby, E. A., \& Joiner, T. E. (2010). The interpersonal theory of suicide. Psychological Review, 117, 575-600.

Van Orden, K. A., Witte, T. K., Gordon, K. H., Bender, T. W., \& Joiner, T. E. (2008). Suicidal desire and the capability for suicide: Tests of the interpersonal psychological theory of suicidal behavior among adults. Journal of Consulting and Clinical Psychology, 76, 72-83.

Watson, J. B., \& Rayner, R. (1920). Conditioned emotional reactions. Journal of Experimental Psychology, 3, 1-14.

Williams, K. D., Cheung, C. K., \& Choi, W. (2000). Cyberostracism: Effects of being ignored over the Internet. Journal of Personality and Social Psychology, 79, 748-762.

World Health Organization. (2014a). Mental health-Suicide prevention. Geneva, Switzerland. Retrieved August 1, 2014, from http:// www.who.int/mental_health/prevention/suicide/suicideprevent/en/

World Health Organization. (2014b). WHO mortality database. Retrieved November 14, 2014, from http://apps.who.int/healthinfo/ statistics/mortality/whodpms/

Yip, P. S. F., \& Cheung, Y. B. (2006). Quick assessment of hopelessness: A cross-sectional study. Health and Quality of Life Outcomes, 4, 13. doi: 10.1186/1477-7525-4-13

Young, E. E., Lariviere, W. R., \& Belfer, I. (2012). Genetic basis of pain variability: Recent advances. Journal of Medical Genetics, 49, 1-9. 